\title{
The immunoglobulin $\mathrm{CH} 1$ constant region modulates antigen binding affinity and functional activities of the broadly neutralizing 2F5 HIV specific antibody
}

\author{
Daniela Tudor ${ }^{1 *}$, Anne-Sophie Drillet ${ }^{1}$, Isabelle Schwartz-Cornil ${ }^{2}$, Ruizhong Shen ${ }^{3}$, Phillip D Smith ${ }^{3}$, \\ Morgane Bomsel ${ }^{1}$
}

From $16^{\text {th }}$ International Symposium on HIV and Emerging Infectious Diseases

Marseille, France. 24-26 March 2010

\section{Background}

The ability of the heavy chain constant region $(\mathrm{CH})$ to affect antibody affinity and specificity could be at the origin of a stronger or weaker memory response, depending on the isotype. Using as a model the broadly neutralizing human $\mathrm{mAb} 2 \mathrm{~F} 5$, directed against the membrane proximal region (MPER) of the HIV-1 envelope transmembrane subunit gp41, we investigated the interplay between 2F5 isotype and functional activity.

\section{Methods}

A 2F5 IgA isotype was constructed from the 2F5 IgG1. Functional monomeric 2F5 IgA and IgG1 were expressed in $\mathrm{CHO}$ cells and their immunochemical characteristics and anti-HIV-1 in-vitro activity were evaluated.

\section{Results}

As compared to 2F5 IgG1, 2F5 IgA sharing identical VH and VL domains but in a different $\mathrm{CH}$ context: (i) binds with higher affinities gp41 and MPER peptides; (ii) has an increased capacity at inhibiting endocytosis of HIV-1 by dendritic cells; (iii) has an increased HIV-1 neutralizing activity in lymphocytic CD4+ T cells; (iv) blocks more efficiently HIV-1 transcytosis across epithelial monolayers in-vitro and normal human rectal mucosa, but (v) has lower ADCC activity. Epitope mapping with a 7 mer epitope library shows that 2F5 IgA recognizes essentially the same hexapeptide epitope as its IgG counterpart.

\section{Discussion}

These results show that the $\mathrm{CH}$ region can fine-tune the specificity of an antibody, by modulating its binding affinity to the antigen and the neutralizing activity of variable-region of otherwise identical antibodies. The determinant role of $\mathrm{CH}$ region on affinity and specificity changes our understanding of vaccine responses. In the context of HIV-1, which is mainly transmitted sexually, these results strongly suggest that raising a mucosal humoral IgA based response will be superior to an IgG one in blocking HIV-1 transmission.

\section{Author details \\ ${ }^{1}$ Institut Cochin, Paris, France. ${ }^{2}$ INRA, Jouy-en-Josas, France. ${ }^{3}$ University of Birmingham, Alabama, USA.}

Published: 11 May 2010

\section{doi:10.1186/1742-4690-7-S1-P23}

Cite this article as: Tudor et al:: The immunoglobulin $\mathrm{CH} 1$ constant region modulates antigen binding affinity and functional activities of the broadly neutralizing 2F5 HIV specific antibody. Retrovirology 2010 7(Suppl 1):P23.

* Correspondence: daniela.tudor@inserm.fr

${ }^{1}$ Institut Cochin, Paris, France 